\title{
Optimising Carbon Electrode Materials for Adsorptive
}

\section{Stripping Voltammetry}

Korbua Chaisiwamongkhol ${ }^{\mathrm{a}}$, Christopher Batchelor-McAuley, Stanislav V. Sokolov ${ }^{\mathrm{a}}$, Jennifer Holter ${ }^{\mathrm{b}}$, Neil P. Young ${ }^{\mathrm{b}}$, Richard G. Compton ${ }^{\mathrm{a} *}$

aDepartment of Chemistry, Physical \& Theoretical Chemistry Laboratory, University of Oxford, South Parks Road, Oxford, OX1 3QZ, United Kingdom

${ }^{b}$ Department of Materials, University of Oxford, Parks Road, Oxford, OX1 3PH, United Kingdom

*corresponding author: Richard G. Compton, Department of Chemistry, Physical \& Theoretical Chemistry Laboratory, University of Oxford, South Parks Road, Oxford, OX1 3QZ, United Kingdom

Email: richard.compton@ chem.ox.ac.uk. Tel: +44(0)1865275957 Fax: +44(0)1865275410 


\begin{abstract}
Different types of carbon electrode materials for adsorptive stripping voltammetry are studied through the use of cyclic voltammetry. Capsaicin is utilised as a model compound for adsorptive stripping voltammetry using unmodified and modified basal plane pyrolytic graphite (BPPG) electrodes modified with multi-walled carbon nanotubes, carbon black or graphene nanoplatelets, screen printed carbon electrodes (SPE), carbon nanotube modified screen printed electrodes, and carbon paste electrodes. We compare the analytical performance of the different electrodes in terms of sensitivity and limit of detection, concluding that carbon electrodes modified with high surface area material exhibit an improvement of sensitivity. In terms of limit of detection, increasing the electrode surface area however does not improve the detection limit; the limit of detection is similar for all electrodes studied.
\end{abstract}

Keywords: adsorptive stripping voltammetry, carbon electrodes, modified carbon electrodes, sensitivity, limit of detection 


\section{Introduction}

The adsorptive stripping voltammetry technique (AdsSV) has found widespread application in the analytical detection and quantification of a broad range of target analytes [1-5]. It involves the accumulation of target compound(s) onto the surface of an electrode via adsorption as a first step. Once a significant surface coverage has been attained, a potential sweep is performed leading to the oxidation or reduction of the adsorbed analyte(s). Measurement of the charge (or current) from the stripping peak along with suitable calibration gives rise to the quantification of the adsorbate in bulk solution [6]. The electrochemical response of the surface-bound species is thus expected to be highly sensitive to the surface area of the electrode surface and the extent of adsorption.

Carbon electrode materials come in variety of forms and have found extensive application in electrochemical studies [7]. Generally, carbon electrodes possess advantages over other metal electrodes due to their comparatively low cost, wide range of potential windows, and high chemical stability [8]. Well-known carbon allotropes include graphite, graphene, diamond, and fullerenes all of which have been used electrodically. Among these allotropes the most common are based on the graphite structure. Surface treatment and modification are methods of considerable interest at present because they offer the chance to increase surface roughness, surface area or oxygenated functional groups on the electrode surface. Methods of surface treatment include electrochemical [9], thermal treatment [10], microwave-assisted activation [11], plasma treatment [12], and modification with high surface area materials [13]. Some of the aforementioned pre-treatment carbon surface methods may lead to more unreproducible results and is outside of scope of present work which focuses on carbon electrode materials. In this context, the modification of the surface of electrode with high surface area materials is attractive. Since the several breakthrough discoveries in the synthesis and fabrication of nanocarbon materials in the last few decades, 
there has been increased interest in several forms of these materials including single-walled and multi-walled carbon nanotubes [14, 15], carbon black [16], nanodiamond [17-19], nanohorns $[20,21]$, and graphene $[22,23]$. The AdsSV technique has been reported through the use of different types of carbon based electrodes in applications of electroanalysis which include carbon materials mentioned above and carbon paste [24-26], as well as screen printed electrodes [3, 27-29]. Carbon nanotubes, rediscovered in 1991 by lijima [30], are made from a graphite sheet rolled seamlessly, producing a tube diameter 1-2 $\mathrm{nm}$ called singlewalled nanotubes (SWCNTs) whereas multiwalled carbon nanotubes are comprised of several tubes inside each other having diameter varying from 2 to $50 \mathrm{~nm}$ [31]. Carbon black or nanocarbon composes of approximately spherical carbon particles, which typically forms aggregates of 10 or more spheres [32]. Various synthetic routes can result in variable sizes of carbon black, with primary particles available with nanodimensions [33]. Graphene nanoplatelets belong to the family of graphene materials which exhibit the advantageous properties of graphene including large surface-to-volume ratio and high electron mobility but avoid the poor stability of graphene which agglomerates or restacks via van der Waals interactions [22, 34-36]. These materials are multilayer particles typically comprising 10-30 graphene sheets [37].

As mentioned carbon paste electrodes are also widely used for the AdsSV study. Carbon paste electrode (CPE) in which carbon is mixed with a liquid binder to form electrodes was first reported by Adams in 1958 [38]. After the preliminary report published by Adams, a huge variety of applications have demonstrated the wide spread applicability of carbon paste electrode in the field of electrochemical analysis [26, 39]. Carbon paste electrodes are interesting due to the ease of their preparation, low background currents (compared to graphite or noble metal electrodes), easily renewed surface, and the feasibility of incorporating different substances into the paste preparation [40]. 
The basic requirements for the development of electroanalytical methods include high sensitivity, low detection limit, cost effectiveness as well as ease of use. The electrochemical response from AdsSV is highly dependent on the electrode surface area, as a higher surface area often leads to an improvement of sensitivity. However, in terms of analysis the detection limit is also an important parameter. In voltammetric experiments, the detection limit is sensitive to both the magnitude of the faradaic and background capacitative currents.

Because of the importance of capacitance on the limit of detection, the background capacitance is as important as the Faradaic signal when assessing different electrodes. Capacitance originates from the double layer charging of an electrode and is proportional to the electroactive area of the electrode [41]. The double layer capacitance of an electrode can be simply and easily estimated from cyclic voltammograms recorded as a function of scan rate. The charging of the electrodes leads to the capacitive current obtained from the difference between the current measured from the forward and back scans. The relationship between the difference of the capacitive currents $(\Delta \mathrm{i})$ and scan rates $(v)$ can be expressed as $\Delta i=2 A C v$

where $\mathrm{A}$ is the effective surface area of the electrode and $\mathrm{C}$ is the electrode capacitance [42]. The relationship from equation 1 predicts a linear plot of capacitive current against scan rate. Accordingly the capacitance can be calculated from the slope of such plots.

Kachoosangi et al. reported work on the adsorptive stripping voltammetry utilising multiwalled carbon nanotube (MWCNT) modified basal plane pyrolytic graphite (BPPG) electrode and commercial MWCNT modified SPEs for detection of capsaicin [43]. Capsaicin compound contains the 2-methoxyphenol moiety where electrochemical oxidation/reduction of capsaicin occurs. There are several molecules containing this 2-methoxyphenol moiety structural motif for instance vanillin [44], curcuminoids [45], gingerol [46], and hesperidin 
[47]. In this paper, we study the adsorptive stripping voltammetry of capsaicin using unmodified and modified basal plane pyrolytic graphite (BPPG) electrodes, screen printed carbon electrodes (SPEs), carbon nanotube modified screen printed electrodes, and carbon paste electrodes. The BPPG electrode is modified using multiwalled carbon nanotubes (MWCNT), carbon black (CB), or graphene nanoplatelets (GNP) by drop-casting. Capsaicin is selected as a model system as it is generically representative of large organic molecules which are amenable to study in adsorptive stripping voltammetry on carbon electrodes and, as such, may guide the establishment of general principles. We compare the AdsSV electroanalytical detection sensitivity and limit of detection from these different electrodes and also consider the influence of electrode capacitance. Our aim is to offer guidance in the choice of type of carbon electrode for adsorptive stripping voltammetry, using the capsaicin system as a model.

\section{Experimental}

\subsection{Chemicals and Apparatus}

Capsaicin (8-methyl-N-vanillyl-6-nonenamide) was purchased from Sigma (Lancashire, UK). All solutions were prepared with deionised water of resistivity not less than $18.2 \mathrm{M} \Omega \mathrm{cm}$ at $298 \mathrm{~K}$ (Millipore, USA). The bamboo-like multiwalled carbon nanotubes, b-MWCNTs $(30 \pm 10 \mathrm{~nm}$ diameter, 5-20 $\mu \mathrm{m}$ length, $>95 \%$ purity) were purchased from NanoLab (Brighton, MA, USA). Graphene nanoplatelets (GNP, $15 \mu \mathrm{m}$ wide, $6-8 \mathrm{~nm}$ thick) 
were purchased from Strem Chemicals, MA, USA. Nanocarbon (diameter $27 \mathrm{~nm} \pm 10 \mathrm{~nm}$, Monarch 430) was purchased from Cabot Performance. Mineral oil was purchased from Aldrich. The Britton-Robinson buffer, $0.05 \mathrm{M}$ at $\mathrm{pH} 1.8$, was prepared using boric acid (99.5\% purity, Aldrich), phosphoric acid (99.99\% purity, Aldrich) and acetic acid (99.5\% purity, BDH).

A basal plane pyrolytic graphite electrode (BPPGE; $4.7 \mathrm{~mm}$ diameter; Le Carbone Ltd, Sussex, UK) was used as a substrate for the modified electrode. A drop-casting method was used for the modification of the electrode by casting $20 \mu \mathrm{L}(4 \times 5 \mu \mathrm{L}$ aliquots $)[48,49]$ of materials of interest dispersed in acetone $(1 \mathrm{mg} / 1 \mathrm{~mL})$ and then allowing the solvent to evaporate. Note that to prevent any possible evaporation of the solvent in suspensions, all of the suspensions were kept in a fridge over the time range of the experiments.

Carbon paste electrodes were prepared by mixing carbon black (diameter $27 \mathrm{~nm} \pm 10$ nm, Monarch 430, Cabot Performance) with mineral oil in a ratio of 3:2 using a pestle and mortar. The well-mixed paste was then packed in a holder made from a Teflon rod with a 3 $\mathrm{mm}$ deep cavity and $4.9 \mathrm{~mm}$ diameter. A copper wire (not exposed to the solution) made electrical contact with the paste.

\subsection{Analytical procedure}

Electrochemical experiments were performed using a three electrode system in a Faraday cage held at $25{ }^{\circ} \mathrm{C}$ using a commercial potentiostat, PGSTAT 101 (MetrohmAutolab, Netherlands). The electrochemical cell was completed using a saturated calomel electrode, SCE, as the reference electrode (SCE +0.244 V vs. SHE, BASi Inc., Japan) and a platinum mesh $99.99 \%$ (Goodfellow, UK) as the counter electrode. 
Experiments were conducted by first immersing the electrode (unmodified BPPG electrode, modified BPPG electrode and CPE) in standard solutions of capsaicin with concentration in the range $0.5-60 \mu \mathrm{M}$. The accumulation of capsaicin was performed under open-circuit potential conditions with stirring of the solution using a stirrer for 1 minute. After adsorption of the analyte, the electrode was transferred to a blank Britton-Robinson buffer solution of $\mathrm{pH} 1.8$ before commencing the voltammetric scanning step.

Commercially available carbon and carbon nanotube screen printed electrodes with a geometric working electrode area of $4 \mathrm{~mm}$ in diameter, a built-in carbon counter electrode and an Ag reference electrode were purchased from Dropsens (C-SPE (DRP-110 Lot: E0013685) and CNT-SPE (DRP-110CNT-160204)). For screen printed electrodes, the accumulation step was performed by dropping $60 \mu \mathrm{L}$ of a desired capsaicin concentration onto the SPEs for 1 minute. The electrode was then rinsed with deionised water after adsorption step, after which the electrode was allowed to thoroughly dry. A $0.05 \mathrm{M}$ BrittonRobinson buffer was dropped onto the electrode surface for the voltammetric experiments [43].

\section{Results and Discussion}

\subsection{Characterisation of commercial screen printed electrodes (SPE) and MWCNT}

Prior to their use, the morphology of (a) the commercial carbon screen printed electrode (C-SPE), (b) the carbon materials used to modify their surfaces of C-SPE (CNTSPE) and (c) the drop casted MWCNT on C-SPE were characterised using a JEOL JSM6500F Scanning Electron Microscope with an accelerating voltage of $5 \mathrm{kV}$ using a secondary electron imaging mode. The carbon materials on the C-SPE, Fig. 1A, showed a dense deposit of a nanostructured surface, whereas the CNT-SPE (Fig. 1B) revealed a woven mesh-like of carbon nanotubes. The CNT-SPE shows a more structured surface which may offer a larger 
effective electrochemical surface area. To compare the morphology of carbon nanotubes on commercial screen printed electrodes and drop-cast modified electrode, MWCNT was dropcasted on the C-SPE which was again imaged using SEM. In Fig. 1C, the SEM image of drop-cast MWCNT shows that multiwalled carbon nanotubes bundle together randomly and possess a larger tubular structure compared to the nanotubes on the CNT-SPE. Note that the characterisation of graphene nanoplatelets (GNP) and carbon black (CB) are shown in supporting information (SI1).

\subsection{Cyclic voltammetric response of capsaicin}

As we use capsaicin system as a model system, we first illustrate and characterise the voltammetric response of capsaicin. Fig. 2 depicts an example of a characteristic voltammetric response of capsaicin at a carbon electrode obtained using AdsSV. Capsaicin was adsorbed on the MWCNT-BPPG electrode from a $30 \mu \mathrm{M}$ solution under open-circuit conditions for 1 minute. Subsequently the voltammetric response of the electrode was recorded at $100 \mathrm{mV} \mathrm{s}^{-1}$. In the first scan, the electrochemical oxidation of the 2methoxyphenol moiety in capsaicin is coupled to an irreversible chemical step to form a molecule containing an ortho-benzoquinone unit as shown in the mechanism inset in Fig. 2. Subsequently in the second scan peaks II and III are observed and correspond to the reversible redox process of the ortho-benzoquinone and catechol couples (C and D inset of Fig. 2) [43]. Qualitatively similar responses were seen from all the electrodes studied except where discussed. 


\subsection{Comparison of the analytical performance of each electrode}

In order to compare the analytical performance of each electrode, we conducted adsorptive stripping voltammetry of capsaicin adsorbed from solutions of various concentrations. For unmodified BPPG electrodes, modified BPPG electrodes, and CPE, the experiments were performed by immersing the electrode of interest in capsaicin standard solutions with differing concentrations in the range 0.5-60 $\mu \mathrm{M}$. The accumulation of capsaicin was performed under open-circuit potential conditions with stirring of the solution for 1 minute. Thereafter, the electrode was transferred to the buffer solution $\mathrm{pH} 1.8$ before commencing the voltammetric scanning step. This procedure was slightly modified for the SPEs, where $60 \mu \mathrm{L}$ of the desired capsaicin concentration in the range of 1-30 $\mu \mathrm{M}$ was dropped onto the SPEs and left for 1 minute. Subsequently, the electrode was rinsed with deionised water after accumulation after which the electrode was completely dried before dropping a $0.05 \mathrm{M}$ Britton-Robinson buffer solution of $\mathrm{pH} 1.8$ onto the electrode. The voltammetric responses of each electrode at $100 \mathrm{mV} \mathrm{s}^{-1}$ were subsequently recorded as a function of the capsaicin concentration as depicted in Fig. 3.

The analytical response of each electrode towards capsaicin was determined by integrating the peak area of oxidative peak of first scan. As shown in the insets of each of Figs. 3A-F and the summary comparison in Fig. 3H, the plot of peak area of capsaicin oxidation peak versus capsaicin concentration shows a linear response for each electrode. From this experiment, the sensitivity was obtained from a slope of the relevant calibration plot and the limit of detection was calculated based on $3 \sigma / \mathrm{S}$ where $\sigma$ is the standard deviation and $S$ is the sensitivity of the calibration curve $[50,51]$. The sensitivity and limit of detection of the different electrodes will be discussed later. In Fig. 3A-F, a clear and well-defined oxidative peak is exhibited for all types of electrode. This is not found to be the case for the graphene nanoplatelets modified BPPG electrode (Fig. 3G) as is discussed below. 
The BPPG electrodes and BPPG electrodes modified by MWCNT and CB (Fig. 3B and C) gave a higher magnitude of the signal of the adsorptive stripping voltammetry as compared to an unmodified BPPG electrode (Fig. 3A). As measured from peak areas of the oxidative peak from $10 \mu \mathrm{M}$ capsaicin, the voltammetric signals on the MWCNTs and CB modified BPPG electrodes are approximately 10 times greater than from the unmodified BPPG electrode. Likewise, the screen printed electrode modified with carbon nanotubes (CNT-SPE) provides a slight larger signal which is approximately 4 times larger compared to unmodified screen printed electrode (C-SPE). Carbon paste electrodes (CPEs) also give welldefined peaks; however, there is a limited linear range from 1-10 $\mu \mathrm{M}$ which might be ascribed to the limited uptake ability of the paste at high capsaicin concentrations $[52,53]$. Considering the GNPs modified BPPG electrode, we found that GNP does not adhere well to the electrode surface and was easily lost from the BPPG electrode surface during the accumulation process. As can be seen from Fig. 3G, the voltammetric responses from GNP modified BPPG electrode (red line) showed a higher capacitance as compared to a bareBPPG electrode (black line) indicated that some GNP remained on the electrode surface after drop-casting. However, after the adsorption of capsaicin on the modified electrode (blue line in Fig. 3G) the capacitance decreased and was smaller than expected as compared to the capacitance of the GNP-BPPG electrode before adsorption of capsaicin (red line in Fig. 3G). The oxidative peak with a small magnitude can be observed at the same potential as the other modified BPPG electrode. This observation suggests that capsaicin can adsorb onto the GNP, but is readily lost.

Next, we considered the surface area and capacitance of the different electrodes studied in this work. The surface area of each electrode was reported in terms of measured surface area or the geometric area. For the modified BPPG electrode with different forms of nanocarbon materials, the effective surface area was calculated from mass of material used in 
the experiment and its known size and shape (see Supporting Information, SI2). The geometric area was used for the screen printed electrodes and the carbon paste electrodes by measurement of the diameter of the carbon surface.

In order to determine the capacitance of each electrode, cyclic voltammetry experiments of the electrode without adsorbed capsaicin was conducted in a solution containing only supporting electrolyte (0.05 M Britton-Robinson buffer). Cyclic voltammograms were recorded as a function of scan rates from $25-400 \mathrm{mV} \mathrm{s}^{-1}$. The capacitance was determined using the relation of capacitance and scan rate shown in equation 1. The current amplitude difference $(\Delta \mathrm{i})$ at a potential of $0.6 \mathrm{~V}$ (vs. SCE) was plotted as a function of scan rate (see Supporting Information, SI3). From this experiment, the linear plots were observed passing through the origin and the capacitance of each electrode was calculated from the slope of this plot (Table 1). According to Table 1, the capacitances of the investigated electrodes were found to lie approximately in the range from $2 \pm 0.1$ to $470 \pm 60$ $\mu \mathrm{F}$. The capacitance of MWCNT and CB modified BPPG electrode are found to be $c a$. $470 \pm 60$ and $151 \pm 16 \mu \mathrm{F}$ respectively which are greatly larger than the unmodified BPPG electrodes (ca. 9 $\pm 4 \mu \mathrm{F})$. Similarly, an unmodified SPE (C-SPE) exhibits a smaller capacitance than a CNT modified SPE namely $2 \pm 0.1 \mu \mathrm{F}$ for the former and $12 \pm 3 \mu \mathrm{F}$ for the latter. Carbon paste electrodes show a low capacitance of $6 \pm 2 \mu \mathrm{F}$. In terms of specific capacitance, the capacitance per unit area, for an ideal electrode this is typically in the range of $10-40 \mu \mathrm{F} \mathrm{cm}{ }^{-2}$ where the capacitance is likely primarily dependent on the double-layer [42]. Table 1 shows that the results for the studied electrodes are roughly the same in the range of $6 \pm 1-96 \pm 23 \mu \mathrm{F} \mathrm{cm} \mathrm{cm}^{-2}$. Note that unlike true capacitors, where capacitances are independent of the voltage across them, double layer capacitance is often a function of potential [42]. 
Apart from capacitance and specific capacitance, Table 1 also summarises the surface area, sensitivity, and limit of detection of each type of electrode. Note that for GNP-BPPG electrodes there is no result reported in Table 1 due to the problem of material falling off from the electrode following drop-casting as discussed above. Considering the sensitivity, the MWCNT- and CB- modified BPPG electrodes give a higher sensitivity compared to unmodified BPPG electrode, SPEs and CPE. This distinct high sensitivity when using the MWCNT-BPPG and CB-BPPG electrode is ascribed to the larger surface area of the electrode. It indicates that the higher electrode surface area the greater the current of adsorbed analyte and so the higher sensitivity. Next turning our attention to a screen printed electrode, we compared two types of commercial SPE namely the carbon screen printed electrodes $(\mathrm{C}$ SPE) and carbon nanotubes modified screen printed electrodes (CNT-SPE). The CNT-SPE shows a slightly higher sensitivity than C-SPE. This result suggests that a modified screen printed electrode with a high surface area resulting from the CNT surfaces improves the sensitivity. For carbon paste electrodes, the sensitivity is comparable to that obtained from the CNT-SPEs. Note that as we mentioned earlier about the limit of linear range of carbon paste electrode, sensitivity and limit of detection are calculated from the linear range of 1 to $10 \mu \mathrm{M}$. Next we considered the limit of detection. Most importantly the limit of detection obtained from each electrode did not vary significantly. On the basis that we mentioned earlier, the limit of detection can be improved by increasing the signal-to-background ratio. From the result, a modified electrode with a high surface area material exhibits an enhancement of faradaic signal. However, the increase of surface area of electrode is manifested in the larger background signal which is exhibited as larger capacitance values in Table 1. These observations indicate that the use of high surface area materials also increases the double layer capacitance. Therefore, the limit of detection does not greatly improve, at least for different carbon based electrodes in the media studied. 


\section{Conclusions}

In this work, we have studied the adsorptive stripping voltammetry of capsaicin which is used as a model system to form general principles for the use of unmodified and modified carbon electrodes where large organic molecules are detected. We have evidenced two important findings with implications for the possible use of different types of carbon electrode for adsorptive stripping voltammetry. First, the magnitude of the signal of adsorptive stripping voltammetry for capsaicin on carbon electrode does not depend greatly on the form of carbon used in the experiments. However, it does depend strongly on the surface area of the materials. Second, we found that increasing the electrode surface area does not improve the limit of detection because the high surface area of electrode concurrently enhances both the adsorptive stripping signals (faradaic signals) and the capacitance signal (background). Therefore, increasing the electrode surface area with high surface area materials does not improve the signal-to-background ratio. Thus the limit of detection is not greatly different. In terms of choice of carbon electrode types for adsorptive stripping voltammetry, unmodified and modified BPPG electrode, SPEs and CPE do not exhibit significant differences on the limit of detection. However, in terms of improvement of sensitivity the modified electrodes show the better sensitivity in comparison to the unmodified. It is also possible to suggest in terms of cost effectiveness that carbon black can be proposed as a useful material for modification of electrode which offers similar advantages to multiwalled carbon nanotubes but at close to zero cost [16].

\section{Acknowledgement}

Korbua Chaisiwamongkhol thanks the Royal Thai Government for supporting her DPhil research. The research leading to these results has received partial funding from the European 
Research Council under the European Seventh Framework Programme (FP/2007-2013)/ERC Grant Agreement no. [320403].

\section{References}

[1] J. Wang, S.B. Hocevar, B. Ogorevc, Carbon nanotube-modified glassy carbon electrode for adsorptive stripping voltammetric detection of ultratrace levels of 2,4,6-trinitrotoluene, Electrochemistry Communications 6 (2004) 176-179.

[2] F. Gutiérrez, G. Ortega, J.L. Cabrera, M.D. Rubianes, G.A. Rivas, Quantification of Quercetin Using Glassy Carbon Electrodes Modified with Multiwalled Carbon Nanotubes Dispersed in Polyethylenimine and Polyacrylic Acid, Electroanalysis 22 (2010) 2650-2657.

[3] M. Moreno, A.S. Arribas, E. Bermejo, M. Chicharro, A. Zapardiel, M.C. Rodríguez, Y. Jalit, G.A. Rivas, Selective detection of dopamine in the presence of ascorbic acid using carbon nanotube modified screen-printed electrodes, Talanta 80 (2010) 2149-2156.

[4] H. Kuramitz, S. Miyagaki, E. Ueno, N. Hata, S. Taguchi, K. Sugawara, Binding assay for cholera toxin based on sequestration electrochemistry using lactose labeled with an electroactive compound, Analyst 136 (2011) 2373-2378.

[5] W. Huang, W. Hu, J. Song, Adsorptive stripping voltammetric determination of 4aminophenol at a single-wall carbon nanotubes film coated electrode, Talanta 61 (2003) 411416.

[6] J. Wang, Controlled-Potential Techniques, Analytical Electrochemistry, John Wiley \& Sons, Inc.2006, pp. 67-114.

[7] R.L. McCreery, Advanced Carbon Electrode Materials for Molecular Electrochemistry, Chemical Reviews 108 (2008) 2646-2687. 
[8] N. Yang, G.M. Swain, X. Jiang, Nanocarbon Electrochemistry and Electroanalysis: Current Status and Future Perspectives, Electroanalysis 28 (2016) 27-34.

[9] J. Wang, M. Pedrero, H. Sakslund, O. Hammerich, J. Pingarron, Electrochemical activation of screen-printed carbon strips, Analyst 121 (1996) 345-350.

[10] R. Gusmao, V. Lopez-Puente, I. Pastoriza-Santos, J. Perez-Juste, M.F. Proenca, F. Bento, D. Geraldo, M.C. Paiva, E. Gonzalez-Romero, Enhanced electrochemical sensing of polyphenols by an oxygen-mediated surface, RSC Advances 5 (2015) 5024-5031.

[11] X. He, Y. Geng, J. Qiu, M. Zheng, S. Long, X. Zhang, Effect of activation time on the properties of activated carbons prepared by microwave-assisted activation for electric double layer capacitors, Carbon 48 (2010) 1662-1669.

[12] S.C. Wang, K.S. Chang, C.J. Yuan, Enhancement of electrochemical properties of screen-printed carbon electrodes by oxygen plasma treatment, Electrochimica Acta 54 (2009) 4937-4943.

[13] L. Agüí, P. Yáñez-Sedeño, J.M. Pingarrón, Role of carbon nanotubes in electroanalytical chemistry: A review, Analytica Chimica Acta 622 (2008) 11-47.

[14] B. Rezaei, N. Askarpour, A.A. Ensafi, Adsorptive stripping voltammetry determination of methyldopa on the surface of a carboxylated multiwall carbon nanotubes modified glassy carbon electrode in biological and pharmaceutical samples, Colloids and Surfaces B: Biointerfaces 109 (2013) 253-258.

[15] R.T. Kachoosangi, G.G. Wildgoose, R.G. Compton, Sensitive adsorptive stripping voltammetric determination of paracetamol at multiwalled carbon nanotube modified basal plane pyrolytic graphite electrode, Analytica Chimica Acta 618 (2008) 54-60.

[16] T.W.B. Lo, L. Aldous, R.G. Compton, The use of nano-carbon as an alternative to multiwalled carbon nanotubes in modified electrodes for adsorptive stripping voltammetry, Sensors and Actuators B: Chemical 162 (2012) 361-368. 
[17] A.F. Azevedo, M.R. Baldan, N.G. Ferreira, Nanodiamond Films for Applications in Electrochemical Systems, International Journal of Electrochemistry 2012 (2012) 16.

[18] J.B. Zang, Y.H. Wang, S.Z. Zhao, L.Y. Bian, J. Lu, Electrochemical properties of nanodiamond powder electrodes, Diamond and Related Materials 16 (2007) 16-20.

[19] L. Chi Hian, K.J. Grehan, C.H. Goeting, R.G. Compton, J.S. Foord, F. Marken, Nanodiamond Thin Film Electrodes: Metal Electro-Deposition and Stripping Processes, Electroanalysis 15 (2003) 169-174.

[20] N. Karousis, I. Suarez-Martinez, C.P. Ewels, N. Tagmatarchis, Structure, Properties, Functionalization, and Applications of Carbon Nanohorns, Chemical Reviews 116 (2016) $4850-4883$.

[21] F. Valentini, E. Ciambella, V. Conte, L. Sabatini, N. Ditaranto, F. Cataldo, G. Palleschi, M. Bonchio, F. Giacalone, Z. Syrgiannis, M. Prato, Highly selective detection of Epinephrine at oxidized Single-Wall Carbon Nanohorns modified Screen Printed Electrodes (SPEs), Biosensors and Bioelectronics 59 (2014) 94-98.

[22] M. Pumera, Graphene-based nanomaterials and their electrochemistry, Chemical Society Reviews 39 (2010) 4146-4157.

[23] L. Wang, M. Pumera, Electrochemical catalysis at low dimensional carbons: Graphene, carbon nanotubes and beyond - A review, Applied Materials Today 5 (2016) 134-141.

[24] M.C. Ortiz, J. Arcos, J.V. Juarros, J. Lopez-Palacios, L.A. Sarabia, Robust procedure for calibration and calculation of the detection limit of trimipramine by adsorptive stripping voltammetry at a carbon paste electrode, Analytical Chemistry 65 (1993) 678-682.

[25] A. Radi, Determination of pantoprazole by adsorptive stripping voltammetry at carbon paste electrode, Il Farmaco 58 (2003) 535-539. 
[26] I. Švancara, K. Vytřas, K. Kalcher, A. Walcarius, J. Wang, Carbon Paste Electrodes in Facts, Numbers, and Notes: A Review on the Occasion of the 50-Years Jubilee of Carbon Paste in Electrochemistry and Electroanalysis, Electroanalysis 21 (2009) 7-28.

[27] O. Domínguez-Renedo, M. Calvo, M. Arcos-Martínez, Determination of Lamotrigine in Pharmaceutical Preparations by Adsorptive Stripping Voltammetry Using Screen Printed Electrodes, Sensors 8 (2008) 4201.

[28] Z. Taleat, A. Khoshroo, M. Mazloum-Ardakani, Screen-printed electrodes for biosensing: a review (2008-2013), Microchimica Acta 181 (2014) 865-891.

[29] P. Kanyong, S. Rawlinson, J. Davis, Fabrication and electrochemical characterization of polydopamine redox polymer modified screen-printed carbon electrode for the detection of guanine, Sensors and Actuators B: Chemical 233 (2016) 528-534.

[30] S. Iijima, Helical microtubules of graphitic carbon, Nature 354 (1991) 56-58.

[31] R.R. Moore, C.E. Banks, R.G. Compton, Basal Plane Pyrolytic Graphite Modified Electrodes: Comparison of Carbon Nanotubes and Graphite Powder as Electrocatalysts, Analytical Chemistry 76 (2004) 2677-2682.

[32] A.I. Medalia, F.A. Heckman, Morphology of aggregates-II. Size and shape factors of carbon black aggregates from electron microscopy, Carbon 7 (1969) 567-582.

[33] A.J. Bard, Electroanalytical Chemistry: A Series of Advances Volume 17, Marcel Dekker, New York, 1990.

[34] J. Poon, C. Batchelor-McAuley, K. Tschulik, R.G. Compton, Single graphene nanoplatelets: capacitance, potential of zero charge and diffusion coefficient, Chemical Science 6 (2015) 2869-2876.

[35] T. Gan, S. Hu, Electrochemical sensors based on graphene materials, Microchimica Acta $175(2011) 1$. 
[36] A. Ambrosi, C.K. Chua, A. Bonanni, M. Pumera, Electrochemistry of Graphene and Related Materials, Chemical Reviews 114 (2014) 7150-7188.

[37] A. Nieto, D. Lahiri, A. Agarwal, Synthesis and properties of bulk graphene nanoplatelets consolidated by spark plasma sintering, Carbon 50 (2012) 4068-4077.

[38] R.N. Adams, Carbon Paste Electrodes, Analytical Chemistry 30 (1958) 1576-1576.

[39] K. Kalcher, J.M. Kauffmann, J. Wang, I. Švancara, K. Vytřas, C. Neuhold, Z. Yang, Sensors based on carbon paste in electrochemical analysis: A review with particular emphasis on the period 1990-1993, Electroanalysis 7 (1995) 5-22.

[40] C. Apetrei, I.M. Apetrei, J.A. De Saja, M.L. Rodriguez-Mendez, Carbon Paste Electrodes Made from Different Carbonaceous Materials: Application in the Study of Antioxidants, Sensors (Basel, Switzerland) 11 (2011) 1328-1344.

[41] K. Cinková, M. Clark, S.V. Sokolov, C. Batchelor-McAuley, L. Švorc, R.G. Compton, Improving Limits of Detection. Microdisc versus Microcylinder Electrodes, Electroanalysis (2016), DOI: 10.1002/elan.201600693.

[42] A. Bard, L. Faulkner, Electrochemical Methods: Fundamentals and Applications, John Wiley \& Sons, Inc2001.

[43] R.T. Kachoosangi, G.G. Wildgoose, R.G. Compton, Carbon nanotube-based electrochemical sensors for quantifying the 'heat' of chilli peppers: the adsorptive stripping voltammetric determination of capsaicin, Analyst 133 (2008) 888-895.

[44] T.R. Silva, D. Brondani, E. Zapp, I. Cruz Vieira, Electrochemical Sensor Based on Gold Nanoparticles Stabilized in Poly(Allylamine hydrochloride) for Determination of Vanillin, Electroanalysis 27 (2015) 465-472.

[45] D.M. Wray, C. Batchelor-McAuley, R.G. Compton, Selective Curcuminoid Separation and Detection via Nickel Complexation and Adsorptive Stripping Voltammetry, Electroanalysis 24 (2012) 2244-2248. 
[46] K. Chaisiwamongkhol, K. Ngamchuea, C. Batchelor-McAuley, R.G. Compton, Electrochemical detection and quantification of gingerol species in ginger (Zingiber officinale) using multiwalled carbon nanotube modified electrodes, Analyst 141 (2016) 63216328.

[47] M.J. Sims, Q. Li, R.T. Kachoosangi, G.G. Wildgoose, R.G. Compton, Using multiwalled carbon nanotube modified electrodes for the adsorptive striping voltammetric determination of hesperidin, Electrochimica Acta 54 (2009) 5030-5034.

[48] Y.Y. Chan, A.Y.S. Eng, M. Pumera, R.D. Webster, Assessments of Surface Coverage after Nanomaterials are Drop Cast onto Electrodes for Electroanalytical Applications, ChemElectroChem 2 (2015) 1003-1009.

[49] A.Y.S. Eng, C.K. Chua, M. Pumera, Intrinsic electrochemical performance and precise control of surface porosity of graphene-modified electrodes using the drop-casting technique, Electrochemistry Communications 59 (2015) 86-90.

[50] D.A. Skoog, Fundamentals of Analytical Chemistry, Thomson-Brooks/Cole2004.

[51] Y.C. Lee, Method Validation for HPLC Analysis of Related Substances in Pharmaceutical Drug Products, Analytical Method Validation and Instrument Performance Verification, John Wiley \& Sons, Inc.2004, pp. 27-49.

[52] R. Nissim, R.G. Compton, Absorptive stripping voltammetry for cannabis detection, Chemistry Central Journal 9 (2015) 41.

[53] R. Nissim, R.G. Compton, Introducing absorptive stripping voltammetry: wide concentration range voltammetric phenol detection, Analyst 139 (2014) 5911-5918. 


\section{Figure captions}

Fig. 1 SEM images of (A) a commercial carbon screen printed electrode (C-SPE), (B) a commercial carbon nanotube screen printed electrode (CNT-SPE) and (C) drop-cast multiwalled carbon nanotubes on a carbon screen printed electrode.

Fig. 2 The overlaid first and second cyclic voltammograms for the adsorptive stripping voltammetry of $50 \mu \mathrm{M}$ capsaicin on a MWCNT-BPPG electrode recorded in a $0.05 \mathrm{M}$ Britton-Robinson buffer solution $\mathrm{pH}$ 1.8, after open-circuit accumulation for 1 minute; scan rate $100 \mathrm{mV} \mathrm{s}^{-1}$. Inset: the proposed electrochemical mechanism of capsaicin [43].

Fig. 3 The first scans of the adsorptive stripping cyclic voltammetric responses to increasing capsaicin concentrations of (A) unmodified-BPPG electrode, (B) a drop casted multiwalled carbon nanotubes on BPPG electrode (MWCNT-BPPGE), (C) a drop casted carbon black on BPPG electrode (CB-BPPGE), (D) a carbon screen printed electrode (C-SPE), (E) a carbon nanotubes screen printed electrode (CNT-SPE), (F) a carbon paste electrode (CPE) and (G) a drop casted graphene nanoplatelets on BPPG electrode (GNP-BPPGE) at $100 \mathrm{mV} \mathrm{s}^{-1}$ in a 0.05 M Britton-Robinson buffer $\mathrm{pH}$ 1.8, after open-circuit accumulation for 1 minute. Inset: the corresponding calibration curves plot using oxidation peak. A comparison of the corresponding calibration curves of each electrode is shown in $(\mathrm{H})$. 
Table 1 Summary of the surface area, sensitivity, limit of detection and capacitance of the different types of electrode modification studied

\begin{tabular}{|c|c|c|c|c|c|}
\hline Electrode & $\begin{array}{l}\text { Effective } \\
\text { surface area } \\
\left(\mathrm{cm}^{2}\right) \\
\end{array}$ & $\begin{array}{l}\text { Sensitivity } \\
\left(\mu \mathrm{C} \mu \mathbf{M}^{-1}\right)\end{array}$ & $\begin{array}{l}\text { LOD } \\
(\mu \mathrm{M}) \\
\end{array}$ & $\begin{array}{l}\text { Capacitance } \\
(\boldsymbol{\mu F})^{*}\end{array}$ & $\begin{array}{l}\text { Specific } \\
\text { capacitance } \\
\left(\mu \mathrm{F} \mathbf{c m}^{-2}\right)^{*} \\
\end{array}$ \\
\hline Unmodified BPPG electrode & 0.17 & 0.59 & 0.24 & $9 \pm 4$ & $52 \pm 21$ \\
\hline Drop-casted MWCNT-BPPG electrode ${ }^{[a]}$ & 19 & 4.72 & 0.17 & $470 \pm 60$ & $24 \pm 3$ \\
\hline Drop-casted CB-BPPG electrode ${ }^{[b]}$ & 23 & 5.26 & 0.19 & $151 \pm 16$ & $6 \pm 0.6$ \\
\hline Drop-casted GNP-BPPG electrode ${ }^{[c]}$ & 27 & - & - & - & - \\
\hline Carbon screen printed electrode & 0.13 & 0.04 & 0.20 & $2 \pm 0.1$ & $14 \pm 3$ \\
\hline Carbon nanotubes screen printed electrode & 0.13 & 0.18 & 0.41 & $12 \pm 3$ & $96 \pm 23$ \\
\hline Carbon paste electrode & 0.19 & 0.28 & 0.40 & $6 \pm 2$ & $30 \pm 9$ \\
\hline
\end{tabular}

[a] Multiwalled carbon nanotubes [b] carbon black [c] graphene nanoplatelets $* n=3$

\section{Graphical abstract}

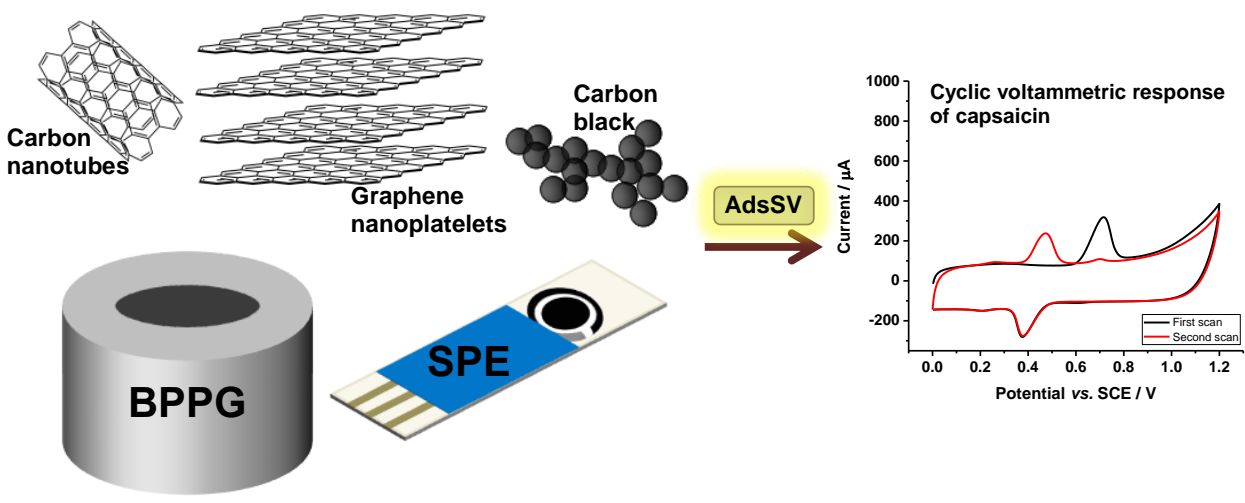

